\title{
Statistical approach to passive target tracking
}

\author{
Melvin J. Hinich and Michael C. Bloom \\ Virginia Polytechnic Institute and State University, Blacksburg, Virginia 24061 \\ (Received 21 August 1980; accépted for publication 14 November 1980)

\begin{abstract}
The paper presents a method for passively tracking a moving target using a sequence of bearings from a surveillance platform. The key assumption for the method is that the target is moving at a constant speed on a fixed heading during the data acquisition period. This is the same assumption that is made for Ekelund ranging. Parameter estimates are computed after bearings are taken as the tracking platforms maneuvers. No specific maneuver by the tracking platform is required. The estimators presented in this paper are approximately maximum likelihood when the target is distant from the platform.
\end{abstract}

PACS numbers: $43.60 . \mathrm{Gk}, 43.60 . \mathrm{Cg}$

\section{INTRODUCTION}

This paper presents a method for estimating the coordinates of a moving target as a function of bearing direction cosines measured from a tracking platform. The parameter estimates are computed after bearing measurements are taken during a period when the tracker maneuvers. One key assumption for the method is that the target is moving at a constant speed on a fixed heading. This assumption is also made for Ekelund ranging. In contrast, our method does not require a specific maneuver by the tracking platform. Another key assumption, which is made for Ekelund ranging, is that the percentage variation in range is small during the observation time. This is a reasonable assumption for a distant target, but it precludes the use of closing tactics.

\section{DEVELOPMENT OF THE TRACKING PARAMETERS}

For a fixed coordinate system, let $x_{T}(t)$ and $x_{B}(t)$ denote the $x$ coordinates of the target and tracker, respectively, at time $t$. Let $y_{T}(t)$ and $y_{B}(t)$ denote their $y$ coordinates. Let $B(t)$ denote the true target bearing at time $t$ measured with respect to the $y$ axis. For example, a target on the $y$ axis would be at $0^{\circ}$ or $180^{\circ}$. Then as seen from Fig. 1,

$$
\sin B(t)=\left[x_{T}(t)-x_{B}(t)\right] / R(t)
$$

and

$$
\cos B(t)=\left[y_{T}(t)-y_{B}(t)\right] / R(t),
$$

where $R(t)$ is the true target range.

Assume that the tracker uses a circular hydrophone array to detect acoustic waves radiating from the target. Bearing information is usually obtained by delayand-sum beamforming. Remember that the direction $\operatorname{cosines} \sin B$ and $\cos B$ are needed to determine the delays used to steer a beam in direction $B$. Suppose that the beam angle that gives maximum signal energy during the integration time is $\hat{B}$. This is the estimate of the target bearing during the integration time. Let $s(t)=\sin B(t), c(t)=\cos B(t)$, and $\hat{s}(t)$ and $\hat{c}(t)$ denote the direction cosines corresponding to $\hat{B}$. They can be obtained from the beamformer's signal processor in theory, but conventional bearing trackers do not output these statistics.
Levin, ${ }^{1}$ and Hinich and Shaman ${ }^{2}$ show that these estimators of $\sin B$ and $\cos B$ are maximum-likelihood estimators if the ambient noise is Gaussian and spatially incoherent. If the array gain is large, moreover, these estimators are approximately Gaussian, unbiased, and independent. For a circular array geometry, the variances of $\hat{s}(t)$ and $\hat{c}(t)$ are equal and are inversely proportional to the energy signal-to-noise ratio (SNR). Expressions for the bearing and direction cosine errors as a function of SNR, aperture, and the number of hydrophones is given by McDonald and Schultheiss, ${ }^{3}$ Clay et al., ${ }^{4}$ and Hinich. ${ }^{5}$

The analytic results presented below do not apply if the direction cosines are estimated by $\sin \hat{B}$ and $\cos \hat{B}$, since they are not independent nor is their variance equal. Our method can be modified to handle these statistics, but our task is to analyze trackers that may be employed in the future and thus we use optimal array processing methods.

Suppose that the tracker estimates $\sin B(t)$ and $\cos B(t)$ at discrete time points $t_{n}=n \tau$, where $\tau$ is the integration time of the beamformer. The statistical expressions derived in the next section are simplified if we set the time origin in the middle of the sampling period, i.e., let $n$ assume integer values $n=-(N-1) / 2, \ldots,(N-1) / 2$ where $N$ is odd. Thus the sampling interval is $(N-1)_{\tau}$. Assume that $\tau$ is selected to ensure that the estimates are uncorrelated over time.

Several important assumptions will now be made about about the target motion and the SNR during the sampling period. First, assume that the target's velocity $v_{T}$ and its heading $\alpha_{T}$ are constant. Thus, for $-(N-1) / 2$, $\ldots,(N-1) / 2$,

$$
x_{T}\left(t_{n}\right)=x_{T}(0)+v_{T} t_{n} \sin \alpha_{T}
$$

and

$$
y_{T}\left(t_{n}\right)=y_{T}(0)+v_{T} t_{n} \cos \alpha_{T}
$$

Second, assume that $v_{T} N \tau \ll R(0)$ and $v_{B} N \tau \ll R(0)$, where $v_{B}$ is the average speed of the tracker. This implies that the range $R(t)$ is approximately constant during the sampling period. Now let $R$ denote the average range in the sampling period. Finally, assume that the SNR varies sufficiently slowly during this period so that the SNR can reasonably be approximated by 


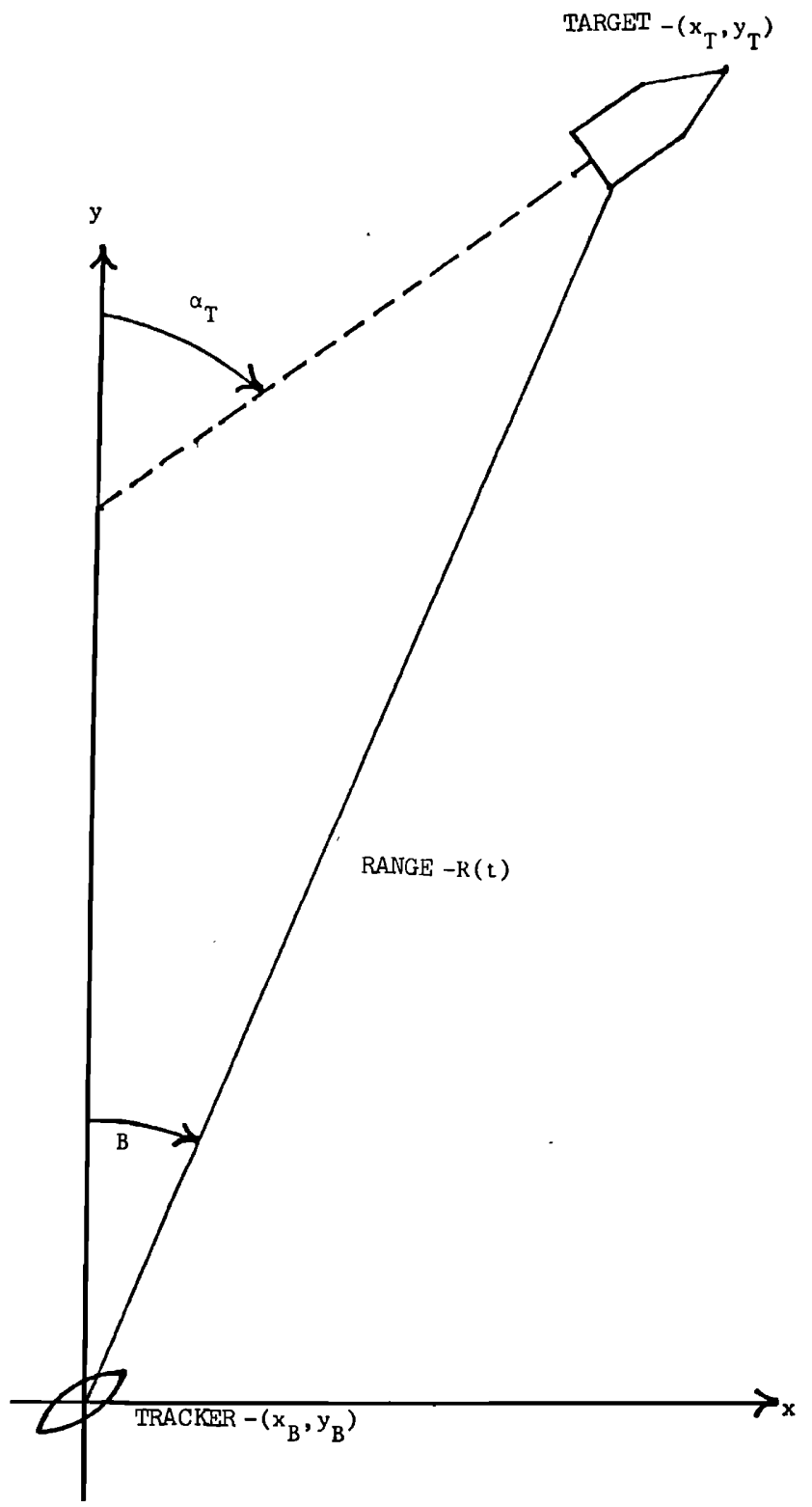

FIG. 1. Platform and target geometry.

a constant. This implies that the variances of the direction cosines are approximately constant. This assumption will be relaxed in a later section.

To simplify notation, select the time unit so that $\tau=1$ and thus $t_{n}=n$. It then follows from (1), (2), and the above assumptions that

$\hat{s}(n)=R^{-1} x_{T}(0)+\left(R^{-1} v_{T} \sin \alpha_{T}\right) n-R^{-1} x_{B}(n)+\epsilon_{s}(n)$,

and

$\hat{c}(n)=R^{-1} y_{T}(0)+\left(R^{-1} v_{T} \cos \alpha_{T}\right) n-R^{-1} y_{B}(n)+\epsilon_{c}(n)$,

where the errors have the following properties (for circular arrays whose gain is large):

(1) $\epsilon_{s}(n)$ and $\epsilon_{c}(n)$ are independent Gaussian random variables with a common variance denoted $\sigma^{2}$,

(2) $\epsilon_{s}(n)$ and $\epsilon_{s}\left(n^{\prime}\right)$ [and $\epsilon_{c}(n)$ and $\epsilon_{c}\left(n^{\prime}\right)$ ] are uncorrelated for all $n \neq n^{\prime}$,

(3) the expected values of these errors are approxi- mately zero if $R(t) \approx R$ during the sampling period.

Now, $\hat{s}(n), \hat{c}(n), x_{B}(n)$ are observed for $n=-(N-1) /$ $2, \ldots,(N-1) / 2$. Hence the coordinates $\left[x_{T}\left(n_{0}\right), y_{T}\left(n_{0}\right)\right]$ of the target at time $n_{0}$ can be estimated.

\section{LEAST-SQUARES ESTIMATES OF TRANSFORMED PARAMETERS}

The maximum-likelihood estimation of these target parameters is facilitated by the following transformations:

$$
\begin{aligned}
& a_{x}=R^{-1} x_{T}(0), \quad a_{y}=R^{-1} y_{T}(0), \\
& \beta_{x}=R^{-1} v_{T} \sin \alpha_{T}, \quad \beta_{y}=R^{-1} v_{T} \cos \alpha_{T},
\end{aligned}
$$

and

$$
b=-R^{-1} \text {. }
$$

Thus from (3)

$$
\hat{s}(n)=a_{x}+\beta_{x} n+b x_{B}(n)+\epsilon_{s}(n),
$$

and

$$
\hat{c}(n)=a_{y}+\beta_{y} n+b y_{B}(n)+\epsilon_{c}(n),
$$

for each $n$. Since the errors are Gaussian and have constant variance, the ordinary least-squares (OLS) estimators of $a_{x}, a_{y}, \beta_{x}$, and $\beta_{y}$ are maximum likelihood. ${ }^{6}$ They also have a joint Gaussian distribution. The maximum-likelihood estimator of $b$ is a weighted average of $\hat{b}_{x}$ and $\hat{b}_{y}$, the OLS estimators of $b$ as computed from (6a) and (6b), respectively. The maximumlikelihood estimators of the target coordinates are functions of these OLS estimators and are computed from the transforms defined in (4). These estimators are presented in Sec. III. Before presenting the weights needed to compute $\hat{b}$, the OLS estimators $\hat{a}_{x}, \hat{\beta}_{x}, \hat{b}_{x}$, and their statistical properties are presented.

The expressions for the OLS estimators of $a_{x}, \beta_{x}$, and $b_{x}$ are simplified if the origin of the coordinate system is placed at the centroid of the platform's track during the sampling period. If this is done, then $\Sigma x_{B}(n)=\Sigma y_{B}(n)=0$. The OLS estimator of $a_{x}$ is

$$
\hat{a}_{x}=N^{-1} \Sigma \hat{s}(n) \text {. }
$$

Its variance is simply

$$
\sigma_{a_{x}}^{2}=N^{-1} \sigma^{2}
$$

Now define $s(n)=\hat{s}(n)-\bar{s}$, where $\bar{s}=N^{-1} \Sigma \hat{s}(n)$, the mean of $\hat{s}(n)$. The OLS estimators $\hat{\beta}_{x}$ and $\hat{b}_{x}$ can be expressed in vector and matrix form as follows:

$$
\left[\begin{array}{l}
\hat{\beta}_{x} \\
\hat{b}_{x}
\end{array}\right]=\left[\begin{array}{cc}
\Sigma n^{2} & \Sigma n x_{B}(n) \\
\Sigma n x_{B}(n) & \Sigma x_{B}^{2}(n)
\end{array}\right]^{-1}\left[\begin{array}{c}
\Sigma n s(n) \\
\Sigma x_{B}(n) s(n)
\end{array}\right] .
$$

The sums are taken from $n=-(N-1) / 2, \ldots,(N-1) / 2$. Thus

$$
\hat{\beta}_{x}=D_{x}^{-1}\left[\Sigma x_{B}^{2}(n) \Sigma n s(n)-\Sigma n x_{B}(n) \Sigma x_{B}(n) s(n)\right],
$$
and

$$
\hat{b}_{x}=D_{x}^{-1}\left[\Sigma n^{2} \Sigma x_{B}(n) s(n)-\Sigma n x_{B}(n) \Sigma n s(n)\right],
$$




$$
D_{x}=\Sigma n^{2} \Sigma x_{B}^{2}(n)-\left[\Sigma n x_{B}(n)\right]^{2} .
$$

From the triangle inequality, $D_{x}=0$ if, and only if, $x_{B}(n)$ is a linear function of $n=t_{n^{*}}$ Thus $D_{x} \neq 0$ if the tracker changes course or speed during the sampling period.

The variance-covariance matrix of $\hat{\beta}_{x}$ and $\hat{b}_{x}$ is

$$
\frac{\sigma^{2}}{D_{x}}\left[\begin{array}{cc}
\Sigma x_{B}^{2}(n) & -\Sigma n x_{B}(n) \\
-\Sigma n x_{B}(n) & \Sigma n^{2}
\end{array}\right] .
$$

Thus the variances of $\hat{\beta}_{x}$ and $\hat{b}_{x}$ are

$$
\sigma_{\beta_{x}}^{2}=\sigma^{2} D_{x}^{-1} \Sigma x_{B}^{2}(n),
$$

and

$$
\sigma_{b_{x}}^{2}=\sigma^{2} D_{x}^{-1} \Sigma n^{2}
$$

It can be shown that $\hat{a}_{x}$ is uncorrelated with $\hat{\beta}_{x}$ and $\hat{b}_{x}$.

It should be obvious from (6a) and (6b) that the OLS estimators $\hat{a}_{y}, \hat{\beta}_{y}$, and $\hat{b}_{y}$ are similar to $\hat{a}_{x}, \hat{\beta}_{x}$, and $\hat{b}_{x}$ with $y_{B}(n)$ and $\hat{c}(n)$ in place of $x_{B}(n)$ and $\hat{s}(n)$. Thus $\hat{a}_{y}$ $=N^{-1} \Sigma \hat{c}(n)$,

$$
\hat{\beta}_{y}=D_{y}^{-1}\left[\Sigma y_{B}^{2}(n) \Sigma n c(n)-\Sigma n y_{B}(n) \Sigma y_{B}(n) c(n)\right],
$$

and

$$
\hat{b}_{y}=D_{y}^{-1}\left[\Sigma n^{2} \Sigma y_{B}(n) c(n)-\Sigma n y_{B}(n) \Sigma n c(n)\right],
$$

where $c(n)=\hat{c}(n)-\bar{c}$ and

$$
D_{y}=\Sigma n^{2} \Sigma y_{B}^{2}(n)-\left[\Sigma n y_{B}(n)\right]^{2} .
$$

Again, $D_{y} \neq 0$ if the tracker makes a course or speed change.

The estimators $\hat{a}_{x}, \hat{\beta}_{x}$, and $\hat{b}_{x}$ are independent of $\hat{a}_{y}$, $\hat{\beta}_{y}$, and $\hat{b}_{y}$ since the errors $\left[\epsilon_{s}(n)\right]$ are independent of $\left[\epsilon_{c}(n)\right]$. It then follows from statistical theory ${ }^{7}$ that $\hat{b}$, the maximum-likelihood estimator of $b$, is the weighted average $\left(\sigma_{b_{x}}^{-2}+\sigma_{b_{y}}^{-2}\right)^{-1}\left(\sigma_{b_{x}}^{-2} \hat{b}_{x}+\sigma_{b_{y}}^{-2} \hat{b}_{y}\right)$. Thus

$$
\hat{b}=\left(D_{x} \hat{b}_{x}+D_{y} \hat{b}_{y}\right) /\left(D_{x}+D_{y}\right)
$$

and its variance is

$$
\sigma_{b}^{2}=\sigma^{2}\left(D_{x}+D_{y}\right)^{-1} \Sigma n^{2} .
$$

From (13) and (19), moreover, the covariance between $\hat{b}$ and $\hat{\beta}_{x}$ is $-\sigma^{2}\left(D_{x}+D_{y}\right)^{-1} \Sigma n x_{B}(n)$ and the covariance between $\hat{b}$ and $\hat{\beta}_{y}$ is $-\sigma^{2}\left(D_{x}+D_{y}\right)^{-1} \sum n y_{B}(n)$.

\section{TARGET RANGE AND COORDINATE ESTIMATES}

Except in some special cases, the variances of the OLS estimators go to zero as $N \rightarrow \infty$. The rate at which this occurs is a function of $N$ and depends upon the form of $\left[x_{B}(n), y_{B}(n)\right]$, i.e., the form of the platform's track. The variances also go to zero as $\sigma^{2}$, the variance of the bearing direction cosine errors, goes to zero. As is shown in the following theorem, the maximum-likelihood estimate of the range is biased if $R \sigma_{b}$ is not small. Assume then that $N$ is sufficiently large and $\sigma$ is sufficiently small so that $R \sigma_{b}$ is small.

Theorem 1. $\hat{R}=-(1 / \hat{b})$ is the maximum-likelihood estimator of $R$. The bias in $\hat{R}$ due to the nonlinear transformation of $\hat{b}$ is

$$
E(\hat{R}-R)=R^{3} \sigma_{b}^{2}+O\left(R^{5} \sigma_{b}^{4}\right),
$$

and its approximate root mean square error is

$$
\operatorname{rmse}(\hat{R})=R^{2} \sigma_{b}+O\left(R^{3} \sigma_{b}^{2}\right),
$$

where $\sigma_{b}^{2}$ is given by (20). Thus the bias is an order of magnitude smaller than the rmse when $R \sigma_{b} \ll 1$.

Proof: $\hat{b}=b+\epsilon_{b}$ is the maximum-likelihood estimator of $b=-R^{-1}$. If $f(b)$ is a continuously differentiable function of $b$, then $f(\hat{b})$ is the maximum-likelihood estimator of $f(b)$. Consequently, $\hat{R}$ is the maximum-likelihood estimator of $R$.

The error $\epsilon_{b}$ is Gaussian $N\left(0, \sigma_{b}^{2}\right)$. Thus $E \epsilon_{b}=E \epsilon_{b}^{3}=0$, $E \epsilon_{b}^{2}=\sigma_{b}^{2}$, and $E \epsilon_{b}^{4}=3 \sigma_{b}^{4}$. Since

$$
\hat{R} / R=1 /\left(1-R \epsilon_{b}\right)=1+R \epsilon_{b}+R^{2} \epsilon_{b}^{2}+R^{3} \epsilon_{b}^{3}+\cdots,
$$

it follows that the expected value of $\hat{R} / R$ is

$$
E(\hat{R} / R)=1+R^{2} \sigma_{b}^{2}+O\left[\left(R \sigma_{b}\right)^{4}\right],
$$

and its mean square error is

$$
\operatorname{mse}(\hat{R} / R)=R^{2} \sigma_{b}^{2}+O\left[\left(R \sigma_{b}\right)^{4}\right] .
$$

Expressions (21) and (22) follow from (24) and (25).

Given $\hat{R}$, the maximum-likelihood estimators of $x_{T}\left(n_{0}\right)$ and $y_{T}\left(n_{0}\right)$ can easily be obtained. Accurate estimates of these target coordinates require that $R \sigma_{b}$ be small.

Theorem 2. The maximum-likelihood estimators of $\left[x_{T}\left(n_{0}\right), y_{T}\left(n_{0}\right)\right]$ are

$$
\hat{x}_{T}\left(n_{0}\right)=\hat{R}\left(\hat{a}_{x}+n_{0} \hat{\beta}_{x}\right),
$$

and

$$
\hat{y}_{T}\left(n_{0}\right)=\hat{R}\left(\hat{a}_{y}+n_{0} \hat{\beta}_{y}\right) .
$$

They are independent. For large $N$, their distributions are approximately Gaussian with zero means and root mean square errors

$$
\begin{aligned}
\operatorname{rmse}\left(\hat{x}_{T}\right) \approx & R \sigma\left(\frac{1}{N}+x_{T}^{2}\left(n_{0}\right) \frac{\Sigma n^{2}}{D_{x}+D_{y}}\right. \\
& \left.-2 n_{0} x_{T}\left(n_{0}\right) \frac{\Sigma n x_{B}(n)}{D_{x}+D_{y}}+n_{0}^{2} \frac{\Sigma x_{B}^{2}(n)}{D_{x}}\right)^{1 / 2},
\end{aligned}
$$

and

$$
\begin{aligned}
\operatorname{rmse}\left(\hat{y}_{T}\right) \approx & R \sigma\left(\frac{1}{N}+y_{T}^{2}\left(n_{0}\right) \frac{\Sigma n^{2}}{D_{x}+D_{y}}\right. \\
& \left.-2 n_{0} y_{T}\left(n_{0}\right) \frac{\Sigma n y_{B}(n)}{D_{x}+D_{y}}+n_{0}^{2} \frac{\Sigma y_{B}^{2}(n)}{D_{y}}\right)^{1 / 2} .
\end{aligned}
$$

Proof: Write $\hat{a}_{x}=a_{x}+\epsilon_{a_{x}}, \hat{\beta}_{x}=\beta_{x}+\epsilon_{\beta_{x}}$, and $\hat{b}=b+\epsilon_{b}$. $\epsilon_{a_{x}}$ and $\epsilon_{B_{x}}$ are uncorrelated Gaussian errors. The error $\epsilon_{b}$ is correlated with $\epsilon_{\beta_{x}}$. Apply (4) and (23) to (26) to obtain the following linear approximation (in the errors):

$$
\begin{aligned}
\hat{x}_{T}\left(n_{0}\right) & \approx R\left(1+R \epsilon_{b}\right) R^{-1} x_{T}(0)+\epsilon_{a_{x}}+n_{0}\left(R^{-1} v_{T} \sin \alpha_{T}+\epsilon_{B_{x}}\right) \\
& =\left(1+R \epsilon_{b}\right)\left[x_{T}\left(n_{0}\right)+R \epsilon_{a_{x}}+R n_{0} \epsilon_{\beta_{x}}\right] \\
& \approx x_{T}\left(n_{0}\right)+R\left[\epsilon_{a_{x}}+x_{T}\left(n_{0}\right) \epsilon_{b}+n_{0} \epsilon_{B_{x}}\right] .
\end{aligned}
$$

Apply (14), (20), and the expression for the covariance 
between $\hat{b}$ and $\hat{\beta}_{x}$ to (27) to derive the expression for rmse $\left(\hat{x}_{T}\right)$. Derive the expression for rmse $\left(\hat{y}_{T}\right)$ in a similar manner.

\section{NONCONSTANT BEARING VARIANCE}

Now relax the restriction that the SNR is constant during the sampling period. There are several methods for eatimating the SNR associated with a specific bearing estimate $\hat{B}$. One method uses output from the beamformer. If the noise field is isotropic in the sector $\hat{B}-\delta<B<\hat{B}+\delta$, then the average of the energy in the beams spanning this $2 \delta$ wide sector (excluding $\hat{B}$ ) is an estimate of the noise field energy. Let $e(\delta)$ denote this estimate. The energy, in the $\hat{B}$ beam is an estimate of signal plus noise. Let $e(\hat{B})$ denote this estimate. Then

$$
\hat{\rho}=e(\hat{B}) / e(\delta)-1 \text {. }
$$

A somewhat more precise estimate uses the average of the coherence between pairs of sensor channels. To illustrate this method, let $\hat{\gamma}^{2}$ denote the average estimated square coherence between two hydrophone channels over the bandwidth of the signal. Then the SNR is estimated by

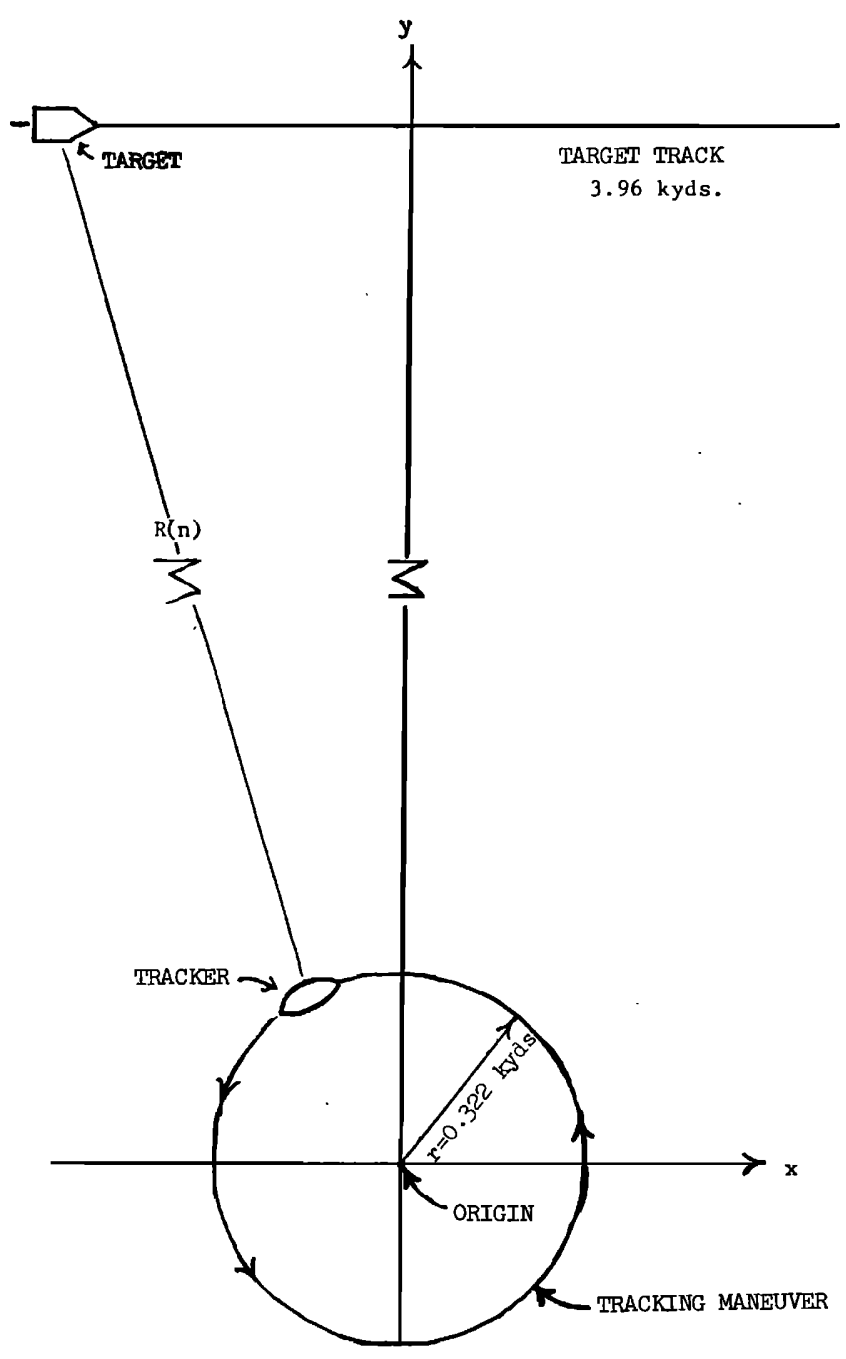

FIG. 2. Simulation geometry.
TABLE I. Results for the Ad Hoc Estimator when $\bar{R}=19.8$ kyds, $\sigma=0.2^{\circ}$. Maximum and minimum range during tracking: 20.181 and 19.485; $\operatorname{rmse}\left(\hat{x}_{T}\right)=0.069, \operatorname{rmse}\left(\hat{y}_{T}\right)=0.667$.

\begin{tabular}{crrrrr}
\hline \hline End of track & Actual & Mean & Mean bias & Stnd. dev. & rmse \\
\hline$\hat{x}_{T}(22)^{\mathrm{a}}$ & 1.980 & 1.970 & -0.011 & 0.092 & 0.092 \\
$\hat{y}_{T}(22)^{\mathrm{a}}$ & 19.800 & 19.785 & -0.015 & 0.091 & 0.092 \\
$\hat{R}_{T}{ }^{\mathrm{a}}$ & 19.836 & 19.816 & -0.020 & 0.895 & 0.895 \\
$\hat{\alpha}_{T}{ }^{\mathrm{b}}$ & 90.000 & 89.828 & -0.172 & 0.549 & 0.575 \\
$\hat{v}_{T}{ }^{\mathrm{c}}$ & 0.360 & 0.359 & -0.001 & 0.017 & 0.017 \\
\hline \hline
\end{tabular}

${ }^{a}$ Distance measured in kiloyards.

${ }^{b}$ Angles measured in degrees.

c Velocities measured in kiloyards/minute.

$$
\hat{\gamma} /(1-\hat{\gamma}),
$$

if the noise field is spatially uncorrelated.

The maximum-likelihood estimators are functions of the variance of the direction cosines if these variances change during the sampling period. Since the variance depends on the SNR, it must be estimated every time a bearing is obtained if one desires to approximate the statistical properties of maximum-likelihood estimators.

The adjustment for a changing error variance is easy using the linear model approach, i.e., use weighted least squares. ${ }^{8}$ Simply divide the independent variables $\left[n, x_{B}(n)\right.$, and $\left.y_{B}(n)\right]$ and the dependent variables $[\hat{s}(n)$ and $\hat{c}(n)$ ] by the estimated variance for each $n$. Since the variance is inversely proportional to the SNR given a narrow-band signal, the adjustment is made by multiply ing the variables by $\hat{\rho}(n)$, a consistent estimator of the SNR at time $t=n \tau$. All the expressions for the estimators and their properties given in the previous sections hold if $n, x_{B}(n), y_{B}(n), \hat{s}(n)$, and $\hat{c}(n)$ are multiplied by $\hat{\rho}(n)$. If the true SNR for each $n$ were known, then the adjusted estimators would be maximum likelihood.

It is important to remember that $\hat{\rho}$ is an estimate of the SNR and not the true SNR for any type of estimator. The error inherent in $\hat{\rho}$ increases the error in the parameter estimates. Consequently, it is generally better to use the ordinary least squares approach rather than weighted least squares (the multiplication adjustment) when the SNR is slowly varying during the sampling period.

TABLE II. Results for the Ad Hoc Estimator when $\bar{R}=19.8$ kyds, $\sigma=0.4^{\circ}$. Maximum and minimum range during tracking: 20.181 and $19.485 ; \operatorname{rmse}\left(\hat{x}_{T}\right)=0.138, \operatorname{rmse}\left(\hat{y}_{T}\right)=1.334$.

\begin{tabular}{crrrrr}
\hline \hline End of track & Actual & Mean & Mean bias & Stnd. dev. & rmse \\
\hline$\hat{x}_{T}(22)^{\mathrm{a}}$ & 1.980 & 1.983 & 0.003 & 0.207 & 0.207 \\
$\hat{y}_{T}(22)^{\mathrm{a}}$ & 19.800 & 19.909 & 0.109 & 0.137 & 0.175 \\
$\hat{R}_{T}{ }^{\mathrm{a}}$ & 19.836 & 19.941 & 0.105 & 2.017 & 2.020 \\
$\hat{\alpha}_{T}{ }^{\mathrm{b}}$ & 90.000 & 89.833 & -0.167 & 1.059 & 1.073 \\
$\hat{v}_{T}{ }^{c}$ & 0.360 & 0.361 & 0.001 & 0.038 & 0.038 \\
\hline \hline
\end{tabular}

a Distance measured in kiloyards.

${ }^{b}$ Angles measured in degrees.

c Velocities measured in kiloyards/minute. 
TABLE III. Results for the Ad Hoc Estimator when $\bar{R}=39.6$ kyds, $\sigma=0.2^{\circ}$. Maximum and minimum range during tracking: 39.951 and $39.281 ; \operatorname{rmse}\left(\hat{x}_{T}\right)=0.138, \operatorname{rmse}\left(\hat{y}_{T}\right)=2.653$.

\begin{tabular}{crrrrr}
\hline End of track & Actual & Mean & Mean bias & Stnd. dev. & rmse \\
\hline$\hat{x}_{T}(22)^{\mathrm{a}}$ & 1.980 & 1.980 & 0.000 & 0.208 & 0.208 \\
$\hat{y}_{T}(22)^{\mathrm{a}}$ & 39.600 & 39.712 & 0.112 & 0.137 & 0.176 \\
$\hat{R}_{T}{ }^{\mathrm{a}}$ & 39.618 & 39.725 & 0.107 & 3.983 & 3.985 \\
$\hat{\alpha}_{T}{ }^{\mathrm{b}}$ & 90.000 & 89.873 & -0.127 & 1.120 & 1.127 \\
$\hat{v}_{T}{ }^{c}$ & 0.360 & 0.361 & 0.001 & 0.037 & 0.037 \\
\hline \hline
\end{tabular}

${ }^{a}$ Distance measured in kiloyards.

${ }^{b}$ Angles measured in degrees.

${ }^{c}$ Velocities measured in kiloyards/minute.

The following ad hoc compromise between ordinary least squares and weighted least squares estimation may provide a more robust estimation method. The compromise rejects a bearing estimate if its estimated SNR is below some threshold value. Suppose, for example, that the SNR for $\hat{s}(2)$ and $\hat{c}(2)$ is below the threshold. Then delete $s(2), c(2), x_{B}(2), y_{B}(2)$, and 2 from the sums in the expressions for $\hat{\beta}_{x}, \hat{\beta}_{y}, \hat{b}_{x}$, and $\hat{b}_{y}$. The target parameters are estimated from those bearings whose SNR exceed the threshold, using the ordinary least squares approach.

\section{SIMULATION}

It is assumed the target is moving at a constant velocity of $90 \mathrm{yds} / 15 \mathrm{~s}$ or $0.36 \mathrm{kyds} / \mathrm{min}$ on a fixed heading. For convenience, the tracker's speed is set at the same rate.

Upon observing the target, the tracker begins a circular tracking maneuver. The radius of the circle is such that the tracker completes two circles during the tracking sequence with a velocity of $0.36 \mathrm{kyds} / \mathrm{min}$. This particular track was chosen for several reasons. First, it is symmetric. That is, one circle is completed in each half of the tracking sequence. Thus the centroid of the tracking sequence is the origin of the coordinate system. Second, the track of two circles yields two estimates of the target's direction cosines at each point where the tracker takes a reading. This enables the OLS routine to more accurately estimate the target's heading since any difference in the two estimates of the target's bearing is due to a change in the target's position. And finally, a circular track minimizes the expected variance of the target's range for

TABLE IV. Results for the Ad Hoc Estimator when $\bar{R}=39.6$ kyds, $\sigma=0.4^{\circ}$. Maximum and minimum range during tracking: 39.951 and $39.281 ; \operatorname{rmse}\left(\hat{x}_{T}\right)=0.276, \operatorname{rmse}\left(\hat{y}_{T}\right)=5.306$.

\begin{tabular}{lrrrrr}
\hline \hline End of track & Actual & Mean & Mean bias & Stnd. dev. & rmse \\
\hline$\hat{x}_{T}(22)^{\mathrm{a}}$ & 1.980 & 2.048 & 0.068 & 0.471 & 0.475 \\
$\hat{y}_{T}(22)^{\mathrm{a}}$ & 39.600 & 41.085 & 1.485 & 0.206 & 1.499 \\
$\hat{R}_{T}{ }^{\mathrm{a}}$ & 39.618 & 41.101 & 1.483 & 9.194 & 9.313 \\
$\hat{\alpha}_{T}{ }^{\mathrm{b}}$ & 90.000 & 89.954 & -0.046 & 2.188 & 2.189 \\
$\hat{v}_{T}{ }^{\mathrm{c}}$ & 0.360 & 0.373 & 0.013 & 0.086 & 0.087 \\
\hline \hline
\end{tabular}

a Distance measured in kiloyards.

${ }^{b}$ Angles measured in degrees.

${ }^{c}$ Velocities measured in kiloyards/minute.
TABLE V. Results for the Ad Hoc Estimator when $\bar{R}=18.1$ kyds, $\sigma=0.2^{\circ}$. Maximum and minimum range during tracking: 19.933 and $16.462 ; \operatorname{rmse}\left(\hat{x}_{T}\right)=0.016, \operatorname{rmse}\left(\hat{y}_{T}\right)=0.505$.

\begin{tabular}{crrrrr}
\hline End of track & Actual & Mean & Mean bias & Stnd. dev. & rmse \\
\hline$\hat{x}_{T}(22){ }^{\mathrm{a}}$ & 0.000 & -0.063 & -0.063 & 0.019 & 0.066 \\
$\hat{y}_{T}(22)^{\mathrm{a}}$ & 16.371 & 18.712 & 2.342 & 0.041 & 2.342 \\
$\hat{R}_{T}{ }^{\mathrm{a}}$ & 18.122 & 18.698 & 0.576 & 0.796 & 0.982 \\
$\hat{\alpha}_{T}{ }^{\mathrm{a}}$ & 150.000 & 86.837 & -63.163 & 1.094 & 63.173 \\
$\hat{v}_{T}{ }^{\mathrm{c}}$ & 0.360 & 0.169 & -0.191 & 0.008 & 0.191 \\
\hline \hline
\end{tabular}

a Distance measured in kiloyards.

${ }^{b}$ Angles measured in degrees.

c Velocities measured in kiloyards/minute.

a smooth track when the tracker has no prior knowledge of the target's heading. We set $N=45$ in our simulations, and thus the radius of the circular track is $0.322 \mathrm{kyds}$. The tracker, therefore, travels $3.96 \mathrm{kyds}$ after having first observed the target taking an observation every $15 \mathrm{~s}$ or every $90 \mathrm{yds}$.

The simulation program calculates $\hat{c}(n)=c(n)+\sigma u_{c}(n)$ and $\hat{s}(n)=s(n)+\sigma u_{s}(n)$ for $n=-22, \ldots, 22$ where $\left[u_{c}(n)\right]$ and $\left[u_{s}(n)\right]$ are computed by the pseudorandom Gaussian $N(0,1)$ generator GGNML in the ISML library. The seed value is advanced after each variate is generated so that the variates are independent. These statistics are used to estimate the range and $\left\{x_{T}(22), y_{T}(22)\right\}$, the target's coordinates at the end of the track, according to the formulae in this paper. Four hundred simulations are made for each set of parameter settings.

At first we set $\alpha_{T}=90^{\circ}$ and centered the track so that $x_{T}(0)=0$ (Fig. 2). After analyzing the results for $R(0)$ $=20 \mathrm{kyds}$ and $\sigma=0$, we discovered that the approximation (6b) for $\cos B$ was poor for this geometry due to insufficient variation in $y_{T}(n)-y_{B}(n)$. The computed values of $-b_{y}$ and $\beta_{y}$ were too small, but $a_{y}$ is accurate. To protect against this misspecification, we modified the estimator of $b$ by selecting the larger of $-\hat{b}_{x}$ and $-\hat{b}_{y}$, which was always $-\hat{b}_{x}$ for the runs summarized in Tables I-IV.

Table I presents the mean, bias, standard deviation, and root mean square error of $\hat{R}=-\hat{b}_{x}^{-1}$ for $\sigma=0.2^{\circ}$ and an average range of $19.8 \mathrm{kyds}$. These results are much better than those for the weighted estimator $-\hat{b}^{-1}$, which has a bias of $17 \mathrm{kyds}$ and a standard deviation of $\mathbf{2 . 3}$ kyds. The weighted estimator consistently overesti-

TABLE VI. Results for the Ad Hoc Estimator when $\bar{R}=18.1$ kyds, $\sigma=0.4^{\circ}$. Maximum and minimum range during tracking: 19.933 and 16.462; $\operatorname{rmse}\left(\hat{x}_{T}\right)=0.032, \operatorname{rmse}\left(\hat{y}_{T}\right)=1.009$.

\begin{tabular}{crrrrr}
\hline \hline End of track & Actual & Mean & Mean bias & Stnd. dev. & rmse \\
\hline$\hat{x}_{T}(22)^{\mathrm{a}}$ & 0.000 & -0.062 & -0.062 & 0.039 & 0.073 \\
$\hat{y}_{T}(22)^{\mathrm{a}}$ & 16.371 & 18.814 & 2.444 & 0.059 & 2.445 \\
$\hat{R}_{T}^{\mathrm{a}}$ & 18.122 & 18.801 & 0.679 & 1.788 & 1.913 \\
$\hat{\alpha}_{T}{ }^{\mathrm{b}}$ & 150.000 & 86.847 & -63.153 & 2.128 & 63.189 \\
$\hat{v}_{T} \mathrm{c}$ & 0.360 & 0.170 & -0.190 & 0.018 & 0.190 \\
\hline \hline
\end{tabular}

${ }^{a}$ Distance measured in kiloyards.

b Angles measured in degrees.

c Velocities measured in kiloyards/minute. 
TABLE VII. Results for the Ad Hoc Estimator when $\bar{R}=37.9$ kyds, $\sigma=0.2^{\circ}$. Maximum and minimum range during tracking: 39.667 and $36.261 ; \operatorname{rmse}\left(\hat{x}_{T}\right)=0.034, \operatorname{rmse}\left(\hat{y}_{T}\right)=2.319$.

\begin{tabular}{crrrrr}
\hline \hline End of track & Actual & Mean & Mean bias & Stnd. dev. & rmse \\
\hline$\hat{x}_{T}{ }^{(22)}{ }^{\mathrm{a}}$ & 0.000 & -0.030 & -0.030 & 0.038 & 0.048 \\
$\hat{y}_{T}{ }^{(22)}{ }^{\mathrm{a}}$ & 36.171 & 38.644 & 2.474 & 0.058 & 2.474 \\
$\hat{R}_{T^{a}}$ & 37.903 & 38.633 & 0.730 & 3.763 & 3.833 \\
$\hat{\alpha}_{T}{ }^{\mathrm{b}}$ & 150.000 & 88.351 & -61.649 & 2.231 & 61.689 \\
$\hat{v}_{T}{ }^{c}$ & 0.360 & 0.176 & -0.184 & 0.019 & 0.185 \\
\hline \hline
\end{tabular}

${ }^{a}$ Distance measured in kiloyards.

b Angles measured in degrees.

c Velocities measured in kiloyards/minute.

mated the range in the runs we made.

In addition to $R, x_{T}(22)$, and $y_{T}(22)$, we estimated $v_{T}$ and $\alpha_{T}$. We used $\hat{\alpha}_{T}=\tan ^{-1}\left(\hat{\beta}_{x} / \hat{\beta}_{y}\right)$ and $\hat{v}_{T}=\left[\hat{R}^{2}\left(\hat{\beta}_{x}^{2}\right.\right.$ $\left.\left.+\hat{\beta}_{y}^{2}\right)\right]^{1 / 2}$. The asymptotic rmse value of $x_{T}$ and $y_{T}$ are also computed.

Table I shows the results when the direction cosine errors are doubled from $0.2^{\circ}$ in Table $I$, to $0.4^{\circ}$. The doubling of the errors approximately doubles the rmse's of all the estimated variables.

Table III presents the simulation results when the range to target is doubled but the bearing errors are $0.2^{\circ}$. With the exception of the estimated range, the rmse's of the estimates are approximately equal to those obtained when the errors were doubled. Increasing either the range to target or the errors on the bearing estimates by a multiplicative constant, $k$, increases the bias by approximately $k$. This empirical relationship does not hold for the estimated range. Although the rmse of the estimated range does approximately double when the bearing errors are doubled, it increases by approximately a factor of 4 when the range to target is doubled.

The calculated rmse values of $\hat{x}_{T}(22)$ and $\hat{R}$ are of the order of magnitude of their theoretical limits. The small rmse values for $\hat{y}_{T}(22), \hat{\alpha}_{T}$, and $\hat{v}_{T}$ are misleading since $\hat{\beta}_{y} \approx 0$ results from the small variation of $y_{T}(n)-y_{B}(n)$, not because $\beta_{y}=0$. The poor fit of $(6 b)$ causes biases when $\alpha_{T} \neq 0$.

To provide some insight into these biases, we made $\alpha_{T}=150^{\circ}$ but started the target at the same position as before. The results for $\sigma=0.2^{\circ}$ and $0.4^{\circ}$ are shown in Tables V-VIII. The estimates of $y_{T}(22), \alpha_{T}$, and $v_{T}$
TABLE VIII. Results for the Ad Hoc Estimator when $\bar{R}=37.9$ kyds, $\sigma=0.4^{\circ}$. Maximum and minimum range during tracking: 39.667 and 36.261; $\operatorname{rmse}\left(\hat{x}_{T}\right)=0.067, \operatorname{rmse}\left(\hat{y}_{T}\right)=4.639$.

\begin{tabular}{lrrrrr}
\hline \hline End of track & Actual & Mean & Mean bias & Stnd. dev. & rmse \\
\hline$\hat{x}_{T}(22)^{a}$ & 0.000 & -0.032 & -0.032 & 0.081 & 0.087 \\
$\hat{y}_{T}{ }^{a}(22)$ & & & & & \\
$\hat{R}_{T}{ }^{a}$ & 36.171 & 39.899 & 3.728 & 0.086 & 3.729 \\
$\hat{\alpha}_{T}{ }^{b}$ & 37.903 & 39.891 & 1.988 & 8.584 & 8.811 \\
$\hat{v}_{T}{ }^{c}$ & 150.000 & 88.503 & -61.497 & 4.405 & 61.654 \\
\hline \hline
\end{tabular}

a Distance measured in kiloyards.

b Angles measured in degrees.

${ }^{c}$ Velocities measured in kiloyards/minute.

are biased but the results for $\hat{R}$ are good.

Further work with more realistic scenarios is needed to determine the value of our approach in future passive tracking systems. Our range estimator gives good results for the simulations we performed.

\section{ACKNOWLEDGMENTS}

This work was begun at the Naval Coastal Systems Center, Panama City, FL under SF 34371691, Sonar and Torpedo Countermeasures Block Program. The rest of the work was supported by the Office of Naval Research (Statistics and Probability Program) under contract. We thank Harold Jarvis for the suggestions he made in his review. His comments helped us, and we appreciate his opennesss.

${ }^{1}$ M. J. Levin, "Least-Squares Array Processing for Signals of Unknown Forms," Radio Electron. Eng. 29, 213-222 (1965).

${ }^{2}$ M. J. Hinich and P. Shaman, "Parameter Estimation for an $\boldsymbol{R}$-Dimensional Plane Wave Observed with Additive Independent Gaussian Errors," Am. Math. Statist. 43, 153-169 (1972).

${ }^{3}$ V. H. MacDonald and P. M. Schultheiss, "Optimum Passive Bearing Estimation," J. Acoust. Soc. Am. 46, 37-43 (1969).

${ }^{4}$ C. S. Clay, M. J. Hinich, and P. Shaman, "Error Analysis of Velocity and Direction Measurements of Plane Waves Using Thick Large-Aperture Arrays," J. Acoust. Soc. Am. 53, 1161-1166 (1973).

${ }^{5}$ M. J. Hinich, "On Errors in Some Papers on Array Processing," J. Acoust. Soc. Am. 65, 530-531 (1979).

${ }^{6} \mathrm{M}$. G. Kendall, The Advanced Theory of Statistics (Hafner, New York, 1951), 3rd ed., Chap. 22.

${ }^{7}$ Ref. 6, Chap. 17.

${ }^{8}$ F. A. Graybill, An Introduction to Linear Statistical Models, Vol. 1 (Wiley, New York, 1961). 